\title{
W-band 5 MW pulse relativistic gyrotron: development and experimental implementation
}

\author{
Alexander N. Leontyev, E.B. Abubakirov, A.V. Chirkov, G.G. Denisov, Yu.M. Guznov, \\ S.Yu. Kornishin, O.P. Plankin, R.M. Rozental, A.S. Sedov, E.S. Semenov, N.A. Zavolsky, \\ S.A. Zapevalov and V.E. Zapevalov \\ Institute of Applied Physics of the Russian Academy of Sciences, Nizhny Novgorod, Russia, jamasiro@mail.ru
}

Powerful sources of the millimeter wavelength range are of considerable interest for a number of applications, such as plasma heating in controlled thermonuclear fusion devices, power supply systems for charged particles linear accelerators with an ultra-high acceleration rate and new radar schemes. The most promising versions of multi-megawatt sources operating in the band seem to be relativistic gyrotrons and gyroklystrons.

A new version of the pulsed relativistic gyrotron operating at the 3-mm wavelength range was developed and tested in experiment. Earlier, significant results were achieved in the realization of centimeter and long-wave millimeter range relativistic gyro-devices in the IAP RAS $[1,2]$. These achievements was based on the detailed study [3], which showed the possibility of obtaining high operation efficiency in gyrotrons at any initial electron energy, including the relativistic one. However, the decrease in the working wavelength, while maintaining the energy parameters of the beam and radiation, entails increasing of the cross-section of the electrodynamic system and, accordingly, the transition to higher-order operating modes. Such a transition makes more complex solving of the key problems of the gyrotron development, first of all mode selection end electron beam formation. Also, an important part of the work was the conjugation of a new short-wave device with elements of the highvoltage electron accelerator already in existence.

Scheme of the experiment. The gyrotron is based on the "Saturn-F" electron accelerator [4], which can generate electron beams with a particle energy of up to $500 \mathrm{keV}$ and a current of up to $200 \mathrm{~A}$ in pulses with the duration and repetition rate being equal to $1 \mu \mathrm{s}$ and 10 pps, respectively. Overall view and the schematic diagram and of the relativistic gyrotron is presented in Fig. 1 and Fig. 2, correspondingly. A helical electron beam is formed by a three-electrode magnetron-injection gun. Theoretical analysis and optimization of the gun was undertaken with the ANGEL code [5]. Calculations showed it feasible to obtain an electron beam at $250 \mathrm{kV}$ of the total voltage and 80-100 A of the beam current with the pitch-factor being equal to 1.3 and an acceptable spread of transverse velocities (20-25\%).

The rotating $\mathrm{TE}_{12,5}$ mode was chosen as the operating one. Single-mode numerical simulations predicted 7-8 MW of the output power with an efficiency of about $35 \%$.

Earlier relativistic gyrotrons were equipped with axial radiation outputs. That approach results, obviously, in very complicated output field structures, corresponding to the operation mode of the gyrotron, and it also has several other drawbacks. The higher-order mode scatters because of the irregularities of the output waveguide, and it results in an increase in the radiation losses.
The new gyrotron was designed with a built-in mode converter, which provides transformation of the operating mode into the Gaussian wave beam and, simultaneously, separation between the regions of propagation of the RF wave and transportation and collection of the exhausted electron beam (Fig. 3). The converter consists of a radiator of the $\mathrm{TE}_{12,5}$ mode into open space, which is formed as a corrugated waveguide with a profiled cut of its edge, feeding a parabolic mirror and two guiding mirrors, and a vacuum boron nitride window. The convertor losses are less than $5 \%$.

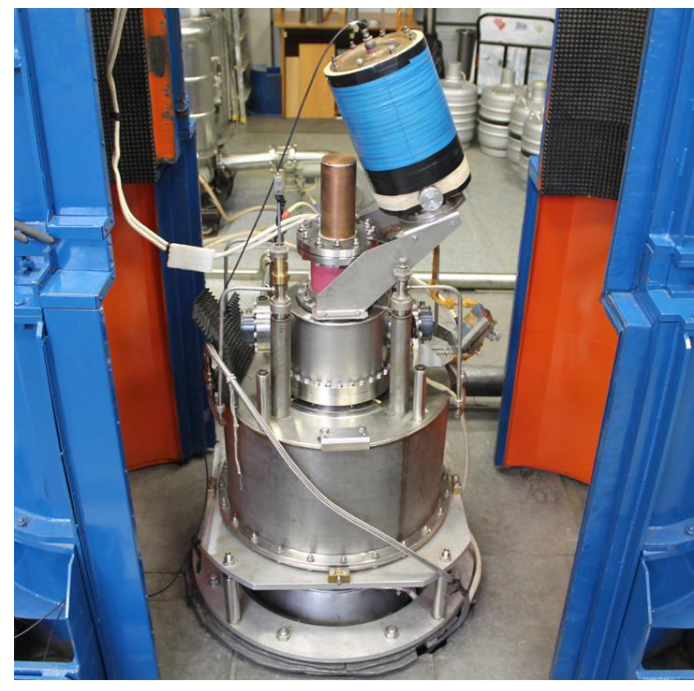

Fig. 1. Gyrotron installation overall view

The measurement system consisted of two sensors for determination of the envelope of the microwave signal, a mixer with a local oscillator to observe the spectrum of radiation, and a calorimetric load to measure the total pulse energy. The energy divided by the pulse duration yielded the peak output power.

Operating regimes. The gyrotron demonstrated stable generation when the magnetic field was set in the vicinity of the cyclotron resonance for the operating mode. The maximum output peak power achieved was 5.6 MW with about $20 \%$ efficiency at the accelerating voltage of $240 \mathrm{kV}$, beam current of $100 \mathrm{~A}$ and leading magnetic field induction of $4.35 \mathrm{~T}$ (Fig. 3). The central frequency was about $94.4 \mathrm{GHz}$ with a spectral width of $3 \mathrm{MHz}$ (Fig. 4).

It was found that the region of the operating magnetic fields was narrower than that predicted theoretically. This seemed to be due to the mode competition, the signs of which were observed in some experiments, where spurious oscillations appeared before and after operation of the working mode (Fig. 5). 


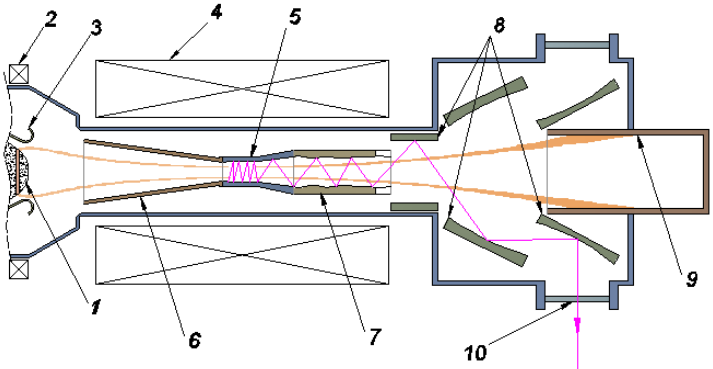

Fig. 2. Schematic diagram of the $94.4 \mathrm{GHz}$ relativistic gyrotron: (1) cathode, (2) cathode coil, (3) anode, (4) cryomagnet, (5) cavity, (6) beam tunnel, (7) working wave radiator, (8) mirrors, (9) collector, (10) output window

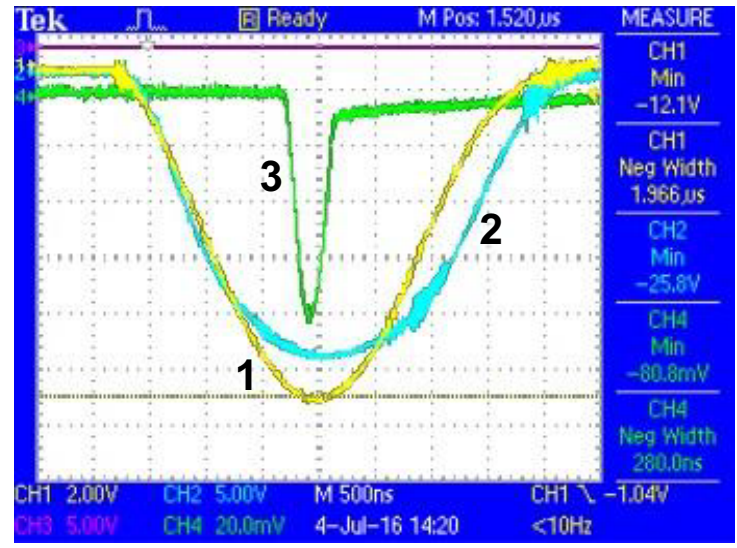

Fig. 3. Oscilloscope traces: (1) waveforms of accelerating voltage (244 kV peak), (2) beam current (103 A peak) and (3) microwave pulse at the regime of maximum output power (5.6 MW). Time scale is $0.5 \mu \mathrm{s} / \mathrm{div}$

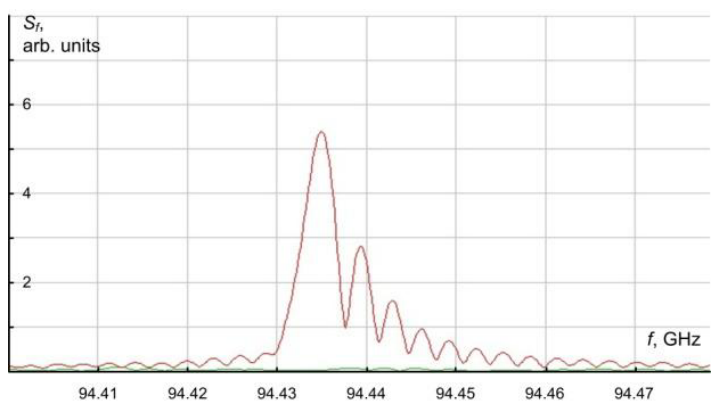

Fig. 4 Spectrum of the microwave pulse shown in Fig. 3. Highfrequency components correspond to the change of the oscillation frequency during the pulse due to variations in the electron energy

Fine adjustment of the voltage and magnetic fields in the resonator and in the cathode area allowed us to obtain a projected oscillation with a stable character; excitation at frequencies differing from the operating one were suppressed or essentially weakened by the main mode generation.
Conclusion. The possibility of obtaining a multimegawatt output power in short-wave gyrotrons is demonstrated. A new version of a pulsed relativistic gyrotron has been developed and experimentally implemented in the 3-mm wavelength range. A stable single-mode generation was realized on the $\mathrm{TE}_{12.5}$ mode of a circular waveguide with subsequent transformation into a Gaussian wave beam. The output power of the gyrotron exceeds 5 MW in pulses of duration about $300 \mathrm{~ns}$.

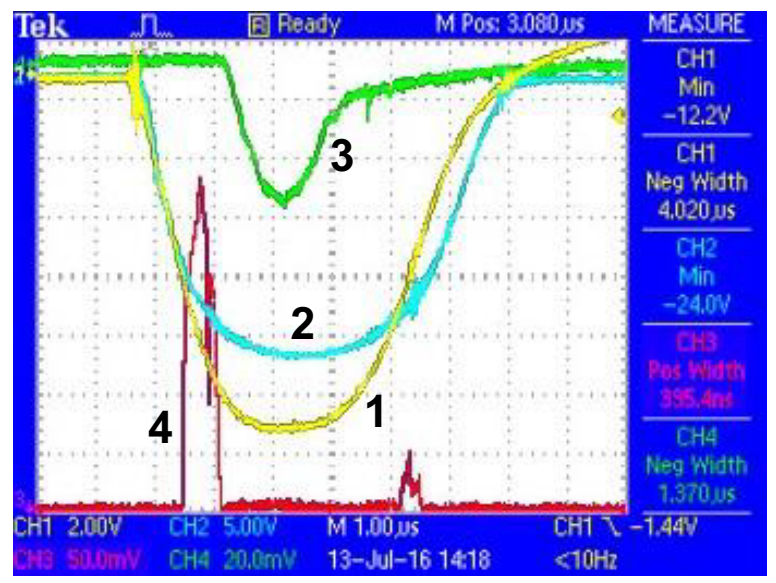

Fig. 5. Waveforms of signals, when parasitic oscillations were detected: accelerating voltage (1); beam current (2); radiation through the main output channel (3) and spurious microwaves registered at the auxiliary vacuum window (4)

\section{References}

1. Zaitsev N.I., Zavolsky N.A., Zapevalov V.E., Ilyakov E.V., Kulagin I.S., Lygin V.K., Moiseev M.A., Nechaev V.E., Petelin M.I., Rozental R.M. Ten-Megawatt Pulsed Gyrotron with a $1-\mathrm{cm}$ Wavelength and a 50\% Efficiency. Radiophysics and Quantum Electronics, 2003, v.46, iss.10, pp.816-819.

2. Zaitsev N. I., Guznov Yu. M., Kuzikov S. V., Plotkin M. E., Tai E. M., Shevchenko A. S. Experimental Investigation of the Relativistic Millimeter-Wave Gyroklystron. Radiophysics and Quantum Electronics, 2014, V. 56, Iss. 8-9, pp. 492-496.

3. Zavolsky N.A., Zapevalov V.E., Moiseev M.A., "Efficiency Enhancement of the Relativistic Gyrotron", International Journal of Infrared and Millimeter Waves, Vol. 22, No. 6, 2001, pp. 813-833.

4. Zaitsev N.I., Zapevalov S.A., Ilyakov E.V., Kornishin S.Yu., Kofanov S.V., Kryltsov M.Yu., Kulagin I.S., Lygin V.K., Malygin A.V., Manuilov V.N., Movshevich B.Z., Perminov V.G., Petelin M.I., Fiks A.Sh., Shevchenko A.S., Tsalolikhin V.I., Kladukhin V.V., Krasnykh A., "500 keV, 200A microsecond electron accelerator with a repetition rate of $10 \mathrm{~Hz}$ ", Proceedings of XXI Russian Accelerator Conference, 2008, Russia, Zvenigorod, pp. 339-341.

5. Plankin O.P., Semenov E.S. Trajectory Analysis of the Electronic-Optical System of Technological Gyrotron. Vestnik NSU. Series: Physics. 2013. V. 8, iss.2, pp.44-54 (in Russian). 